\title{
Spectroscopic Parameters of Neutral Argon Atom
}

\author{
W. SAlah ${ }^{a, *}$ AND O. HassOUnEH ${ }^{b}$ \\ ${ }^{a}$ Department of Physics, The Hashemite University, Zarqa 13115, Jordan \\ ${ }^{b}$ Department of Physics, University of Jordan, Amman 11942, Jordan
}

(Received May 9, 2016; in final form November 23, 2016)

We have calculated the spectroscopic data of the odd series $n d[1 / 2] 1(3 \leq n \leq 9)$ of neutral argon atom relative to the ground level $3 P^{6}{ }^{1} S_{0}$ using a fully relativistic approach based on the Dirac equation. This series is one of three intermediate Rydberg series that converge to the first limit of ionization. The energy levels of this series are predicted. The obtained energy levels results are judged by comparing them with the experimental ones available in the literature. Within the uncertainty in the theoretical results, the agreement between our results and the experimental ones was found to be reasonable. Based on this agreement, the oscillator strengths $f_{i j}$, the radiative transition rates $A_{i j}$, the Landé $g$-factor, the magnetic dipole moment and the electric quadrupole hyperfine constants for the levels in question are calculated. The obtained results of $f_{i j}, A_{i j}$, and Landé $g$-factor are compared with the theoretical and experimental results published by other researchers where available as well as those published by NIST. Within the uncertainty in our results, a good agreement is found. Moreover, the studied levels are situated near and under the first limit of ionization where autoionization effects take place.

DOI: 10.12693/APhysPolA.130.1288

PACS/topics: 31.15.ag, 31.15.aj, 31.15.am

\section{Introduction}

The spectra of highly excited states of the argon atoms have been extensively studied using both conventional photo-absorption and laser excitation techniques [1-3]. These highly excited states, consisting of an external Rydberg electron interacting with anisotropic ionic inner core electrons ( $p^{5}$ configuration) straddle an interesting place in physics. Spectroscopic parameters such as Landé $g$-factors $[2,4-6]$, radiative lifetime and transition probability [7] of these atoms, are required in the analysis of atomic structure, ionization spectroscopy, and its applications. Such parameters of low lying levels have been extensively measured using the Zeeman effect [2]. However, fewer measurements have been taken for highly-lying levels, in particular for levels situated near the first limit of ionization $[2,8]$.

The singly ionized argon gases, ground state $3 p^{6}{ }^{1} S_{0}$ yields two odd-parity states $3 p^{5}{ }^{1} P_{3 / 2}$ and $3 p^{5}{ }^{1} P_{1 / 2}$ corresponding to two different values of the total core angular momentum $j_{c}=3 / 2$ and $1 / 2$, respectively. These two values generate two limits of ionization $I_{3 / 2}=$ $127109.9 \mathrm{~cm}^{-1} \pm 0.1 \mathrm{~cm}^{-1}$ and $I_{1 / 2}=128541.8 \mathrm{~cm}^{-1}$ $\pm 0.1 \mathrm{~cm}^{-1}$ to which all the Rydberg series converge [8].

The excited levels are generally designated in the $j l k-$ coupling commonly called the Racah notation $[9,10]$. In this notation, the orbital angular momentum, $l$, of the excited electron couples with the total angular momentum, $j_{c}$, to give the resultant angular momentum $k$. The latter is then coupled to the spin, $s$, of the Rydberg electron to give the total angular momentum $J$. The fine structure levels belonging to the terms $3 p^{5}{ }^{1} P_{3 / 2}$ and $3 p^{5}{ }^{1} P_{1 / 2}$ are designated by $3 p^{5} n l[k]_{J}$ and $3 p^{5} n l^{\prime}[k]_{J}$, respectively. The odd series $n d[1 / 2]_{1}(3 \leq n \leq 8)$,

\footnotetext{
*corresponding author; e-mail: wsalah@hu.edu.jo
}

$4 d^{\prime}[3 / 2]_{1}, 6 d[3 / 2]_{1}$, and $8 d[3 / 2]_{1}$ of the argon atoms are studied experimentally using laser optogalvanic spectroscopy [2]. Moreover, the odd series $n d[3 / 2]_{1}, n d[1 / 2]_{1}$ and $n s[3 / 2]_{1}$ of argon atom are experimentally studied in the vacuum-ultraviolet region by Yoshino up to values of $n=58,34$ and 22, respectively [8]. For values of principle quantum number $n \leq 18$ for the Rydberg series $n d[3 / 2]_{1}$ and $n d[1 / 2]_{1}$ and $n=20$ for $n s[3 / 2]_{1}$ these series are perturbed by the levels $n s^{\prime}[1 / 2]_{1}(5 \leq n \leq 10)$ situated under the first limit of ionization and by the levels $n d^{\prime}[3 / 2]_{1}(3 \leq n \leq 8)$ situated over the first limit of ionization where autoionization effects take place.

The experimental study of the electronic transitions from the starting levels to those situated near the first limit of ionization is difficult because the classical methods of fluorescence detection are inefficient for the very highly excited levels, which, in general quenched either by collisional ionization, or, spontaneously if these levels are auto-ionized. Therefore, it is difficult to analyze experimentally their data. However, the multichannel quantum defect theory (MQDT) analysis [11] performed by several authors $[12,13]$ has furthermore completed the wealth of experimental data gathered in the case of the highly excited levels. Despite the wealth of experimental and theoretical results provided by many researchers in the world, many spectroscopic parameters for the levels situated near the first limit of ionization still unknown and for that we devote this paper.

In this paper, the energy levels, the oscillator strength, the transition rates, the Landé $g$-factor, and the hyperfine structure constants of the odd series $n d[1 / 2] 1(3 \leq n \leq 9)$ relative to $3 p^{6}{ }^{1} S_{0}$ of neutral argon atom will be theoretically calculated. These calculations will be carried out by employing a fully relativistic approach based on the Dirac equation. These parameters are essential for calculating level populations and spectral line for non-local thermodynamic equilibrium plasmas and X-ray lasers. 
2. The relativistic radiative transition rates $A_{f i}$ and the absorption oscillator strength $f_{i f}$

2.1. The relativistic radiative transition rates $A_{f i}$

The relativistic radiative transition rates $A_{\text {if }}$ per second corresponding to transition from the initial state $i$ to the final state $f$ for an electron outside a closed shell $[11,14,15]$ is

$$
A_{f i}=2 \alpha \omega_{i f} \frac{2 j_{i}+1}{2 L+1}\left(\begin{array}{ccc}
j_{f} & L & j_{i} \\
1 / 2 & 0 & -1 / 2
\end{array}\right)^{2}\left|\bar{M}_{i f}\right|^{2},
$$

where the matrix elements $\bar{M}_{i f}$ for a relativistic radiative transition of a single electron multipole operator of order $L$ [15]. These matrix elements are either the integral for electron multipole transitions or the integral for the electron magnetic multipole transitions [15].

These radiative transitions are defined as

$M_{i f}=\left\{\begin{array}{l}M_{i f}^{(e)}+G M_{i f}^{(1)} \text { for electron multipole transitions, } \\ M_{i f}^{(m)} \text { for magnetic multipole transitions, }\end{array}\right.$ where

$$
\begin{aligned}
& M_{i f}^{(m)}=(\mathrm{i})^{l+1} \frac{2 L+1}{\sqrt{L(L+1)}}\left(k_{f}+k_{i}\right) I_{L}^{+}\left(\omega_{i f}\right), \\
& M_{i f}^{(e)}=(\mathrm{i})^{L}\left\{\sqrt { \frac { L } { L + 1 } } \left[\left(k_{i}-k_{f}\right) I_{L+1}^{+}\left(\omega_{i f}\right)\right.\right. \\
& \left.+(L+1) I_{L+1}^{-}\left(\omega_{i f}\right)\right] \\
& \left.\quad-\sqrt{\frac{L+1}{L}}\left[\left(k_{i}-k_{f}\right) I_{L-1}^{+}\left(\omega_{i f}\right)-L I_{L-1}^{-}\right]\right\} \\
& M_{i f}^{(1)}=(\mathrm{i})^{L}\left[\left(k_{i}-k_{f}\right) I_{L+1}^{+}\left(\omega_{i f}\right)+(L+1) I_{L+1}^{-}\left(\omega_{i f}\right)\right] \\
& \quad+\left(k_{i}-k_{f}\right) I_{L-1}^{+}\left(\omega_{i f}\right) L I_{L-1}^{-}+(2 L+1) J_{L},
\end{aligned}
$$

with

$$
\begin{aligned}
I_{L}^{ \pm} & =\int_{0}^{\infty} j_{l}\left(\frac{\omega_{i f}}{c} r\right)\left(P_{n_{i} k_{i}}(r) Q_{n_{f} k_{f}}(r)\right. \\
& \left. \pm Q_{n_{i} k_{i}}(r) P_{n_{f} k_{f}}(r)\right) \mathrm{d} r, \\
J_{L} & =\int_{0}^{\infty} j_{l}\left(\frac{\omega_{i f}}{c} r\right)\left(P_{n_{i} k_{i}}(r) P_{n_{f} k_{f}}(r)\right. \\
& \left.+Q_{n_{i} k_{i}}(r) Q_{n_{f} k_{f}}(r)\right) \mathrm{d} r,
\end{aligned}
$$

where $j_{l}$ is the spherical Bessel function and $G$ in Eq. (2) is the gauge parameter. It takes the value 0 in the Coulomb gauge $[16,17]$ and $\sqrt{\frac{L+1}{L}}$ in the Babushkin gauge [18-20]. In the non-relativistic limit, $G=0$ gives the velocity form of radiation matrix elements, while $G=\sqrt{\frac{L+1}{L}}$ gives the length form. The radial functions $P_{n k}(r)$ and $Q_{n k}(r)$ are the solutions of the coupled Dirac equation for local central field $V(r)$ [21]:

$$
\left(\frac{\mathrm{d}}{\mathrm{d} r}+\frac{\xi}{r}\right) P_{n k}(r)=\alpha\left(\epsilon_{n k}-V_{i}(r)+\frac{2}{\alpha^{2}}\right) Q_{n k}(r),
$$

$$
\left(\frac{\mathrm{d}}{\mathrm{d} r}-\frac{\xi}{r}\right) Q_{n k}(r)=\alpha\left(-\epsilon_{n k}-V_{i}(r)\right) P_{n k}(r)
$$

with

$$
\xi=\left\{\begin{array}{cc}
-\left(j+\frac{1}{2}\right)=-l-1, & j=l+\frac{1}{2} \\
\left(j+\frac{1}{2}\right)=l, & j=l-\frac{1}{2}
\end{array},\right.
$$

where $\alpha$ is the fine structure constant, and $\epsilon_{n k}$ are the energy levels of an electron in a Coulomb field $V(r)$. These energies are defined by the two quantum numbers $n$ and $k$. The principal quantum number $n$ is given by

$$
\begin{aligned}
& n=n^{\prime}+|\xi|=n^{\prime}+k, \quad|\xi|=k, \\
& n^{\prime}=\left\{\begin{array}{cc}
0,1,2,3, \ldots ., & \xi<0, \\
1,2,3, \ldots, & \xi>0 .
\end{array}\right.
\end{aligned}
$$

On the other hand, the radial functions $P_{n k}(r)$ and $Q_{n k}(r)$ must satisfy the boundary conditions

$$
\left.\begin{array}{l}
P_{n k}(r) \\
Q_{n k}(r)
\end{array}\right\} \rightarrow 0 \text { when }\left\{\begin{array}{c}
r \rightarrow 0 \\
r \rightarrow \infty
\end{array}\right.
$$

\subsection{The absorption oscillator strength $f_{i f}$}

The dimensionless absorption oscillator strength $f_{i f}$ for a transition from the initial level $i$ to the final level $f$ are calculated in the single multipole approximation. In this approximation, the interference between different multipoles transitions will not be taken into account, whereas transition rates corresponding to arbitrary multipoles will be taken into account. Under these conditions, the line strength $f_{i f}$ for the transition from initial atomic state function $\Psi_{i}$ to final state function $\Psi_{f}$ induced by a multipole radiation field operator $\widehat{D}_{m}^{(L)}$ of order $L$ is

$$
f_{i f}=\frac{\pi c}{(2 L+1) \omega_{i f}^{2}}\left|\left\langle\Psi_{i}\left|\widehat{D}_{m}^{(L)}\right| \Psi_{f}\right\rangle\right|^{2} .
$$

The last equation can be written in terms of the reduced matrix elements as

$$
f_{i f}=\frac{\pi c}{(2 L+1) \omega_{i f}^{2}} \sum_{\mu \nu} b_{\mu f} b_{i \nu}\left\langle n_{i} k_{i}\left\|\widehat{D}_{m}^{(L)}\right\| n_{f} k_{f}\right\rangle,
$$

where the reduced matrix elements $\left\langle n_{i} k_{i} \| \widehat{D}_{m}^{(L)}|| n_{f} k_{f}\right\rangle$ are given by

$$
\begin{aligned}
& \left\langle n_{i} k_{i}\left|\widehat{d}^{(L)}\right| n_{f} k_{f}\right\rangle=\left(\frac{\left(2 j_{i}+1\right) \omega_{i f}^{2}}{\pi c}\right) \\
& \times(-1)^{j_{i}-\frac{1}{2}}\left(\begin{array}{ccc}
j_{i} & L & j_{f} \\
1 / 2 & 0 & -1 / 2
\end{array}\right)\left|\bar{M}_{i f}\right| .
\end{aligned}
$$

\section{Landé $g$-factor}

\subsection{Problem setup}

The Hamiltonian $\widehat{H}$ describing the interaction of an atom with a uniform magnetic field $\boldsymbol{B}$ has the form

$$
\widehat{H}=\frac{1}{2} \sum_{i} e \widehat{\alpha}_{i} \cdot\left(\boldsymbol{B} \times \boldsymbol{r}_{i}\right),
$$

where the summation is taken over all atomic $i$-th electron, $e$ is the electron charge and $\widehat{\alpha}=\left(\begin{array}{ll}0 & \widehat{\sigma} \\ \widehat{\sigma} & \widehat{0}\end{array}\right)$ is 
a four matrix known as the Dirac operator and $\widehat{\sigma}$ represents the Pauli matrices. For simplicity, we shall drop the index $i$ in the following equations. In terms of the scalar product of magnetic dipole moment spherical tensor of rank 1, $\mu_{q}^{(1)}$, Eq. (17) becomes

$$
\widehat{H}=\frac{1}{2} \sum \mu_{q}^{(1)} \cdot \boldsymbol{B}
$$

with

$$
\sum \mu_{q}^{(1)}=-\sum \mathrm{i} e \sqrt{\frac{8 \pi}{3}} r \widehat{\alpha} \cdot Y_{l q}^{(0)}(\boldsymbol{r}),
$$

where $Y_{l q}^{(0)}(\boldsymbol{r})$ represent the spherical harmonic vector.

The Landé $g$-factor $g_{J}$ is defined by the magnetic dipole moment operator $\widehat{\mu}$ of an atom in the state $\left|J M_{J}\right\rangle$ as

$$
\widehat{\mu}=-g_{J} \mu_{\mathrm{B}} \widehat{J}
$$

where $\mu_{\mathrm{B}}$ is the Bohr magneton.

Since matrix elements of the Hamiltonian given by Eq. (18) can be calculated in the state $\left|J M_{J}\right\rangle$, the matrix elements of the Hamiltonian $\widehat{H}$ are

$$
\langle\widehat{H}\rangle=g_{J} \mu_{\mathrm{B}}\langle\widehat{J} \cdot \boldsymbol{B}\rangle,
$$

then the Landé $g$-factor $g_{J}$ can be written by means of the Wigner-Eckart theorem [14] as

$$
g_{J}=\frac{1}{2 \mu_{\mathrm{B}}} \frac{\left\langle J \| \sum \mu_{q}^{(1)}|| J\right\rangle}{\sqrt{J(J+1)}} .
$$

On the other hand, the quantum electrodynamics correction imposes a correction on the electron $g_{s}$ factor [22]. This correction is given by

$$
g_{s}=2\left(1+\frac{\alpha}{2 \pi}-0.3828\left(\frac{\alpha}{\pi}\right)^{2}+\ldots\right) \approx 2 \times 1.001160,
$$

where $\alpha=\frac{1}{137}$ is the fine structure constant.

The correction to the electron $g_{s}$ factor leads to a correction to the relativistic interaction Hamiltonian by an amount

$$
\Delta \widehat{H}=0.001160 \mu_{\mathrm{B}} \widehat{\beta} \widehat{\alpha} \cdot \boldsymbol{B}
$$

and this leads to a correction to Landé $g$-factor $g_{J}$ by an amount

$$
\Delta g_{J}=0.001160 \frac{\left\langle J\left\|\sum \mu_{q}^{(1)}\right\| J\right\rangle}{\sqrt{J(J+1)}} .
$$

\subsection{Radial matrix elements}

The relativistic eigenfunction for a state denoted in the Dirac notation by $\left|\Gamma_{J} \Pi J M_{J}\right\rangle$ can be expanded in $j j$-coupled configuration state functions which are eigenfunctions of $J^{2}, J_{z}$ and the parity operator $\Pi$ in the multiconfiguration Dirac-Fock method (MCDF) as

$$
\left|\Gamma_{J} \Pi J M_{J}\right\rangle=\sum_{r} C_{r}\left|\gamma_{J} \Pi J M_{J}\right\rangle,
$$

where $\Gamma_{J}$ and $\gamma_{J}$ represent the configuration and any other quantum number required to specify a state, and $C_{r}$ are the expansion coefficients. On the other hand, the configuration state functions are sums of product of four component spin-orbital function named the Dirac wave function. The spin spherical harmonics $\chi_{k m}$ defined in Dirac wave equation can be written in the $L S J$ - coupling as

$$
\begin{aligned}
& \chi_{k m}=\sum_{q} C\left(l \frac{1}{2} j ; m-q q m\right) Y_{l m-q}(\boldsymbol{r}) \\
& \times\left\{\begin{array}{l}
\left(\begin{array}{l}
1 \\
0 \\
0 \\
1
\end{array}\right), \quad q=\frac{1}{2}, \\
q=-\frac{1}{2} .
\end{array}\right.
\end{aligned}
$$

Moreover, an angular recoupling computer program [23] is used in order to reduce the matrix elements $\left\langle J\left\|\sum \mu_{q}^{(1)}\right\| J\right\rangle$ and $\left\langle J\left\|\sum \Delta \mu_{q}^{(1)}\right\| J\right\rangle$ to terms involving single-particle orbitals only

$$
\begin{aligned}
& \left\langle J\left\|\sum \mu_{q}^{(1)}\right\| J\right\rangle=\sum_{a, b} D_{a b}^{(1)}(r s)\left\langle n_{a} k_{a}\left\|\mu^{(1)}\right\| n_{b} k_{b}\right\rangle, \\
& \left\langle J\left\|\sum \Delta \mu_{q}^{(1)}\right\| J\right\rangle=\sum_{a, b} D_{a b}^{(1)}(r s)\left\langle n_{a} k_{a}\left\|\Delta \mu^{(1)}\right\| n_{b} k_{b}\right\rangle,
\end{aligned}
$$

where

$$
\begin{aligned}
& D_{a b}^{(1)} \equiv-\sum(-1)^{\frac{1}{2}} e \sqrt{\frac{8 \pi}{3}} r \widehat{\alpha} \cdot Y_{l q}^{(0)}(\boldsymbol{r}) \delta_{b b^{\prime}} \sqrt{2 j_{a}+1} \\
& \quad \times \sqrt{2 j_{b}+1}(-1)^{a+b+j_{b}+1} \cdot\left\{\begin{array}{ccc}
b & a & j_{a} \\
1 & j_{b} & a^{\prime}
\end{array}\right\}
\end{aligned}
$$

and the \{\} denotes the $6 \mathrm{j}$-symbol.

However, the single particle matrix elements can be reduced into angular factors and radial integral

$$
\begin{aligned}
& \left\langle n_{a} k_{a}\left\|\mu^{(1)}\right\| n_{b} k_{b}\right\rangle= \\
& -\alpha\left(k_{a}+k_{b}\right)\left\langle n_{a} k_{a}\left\|C^{(1)}\right\| n_{b} k_{b}\right\rangle[r]_{n_{a} k_{a} n_{b} k_{b}}, \\
& \left\langle n_{a} k_{a}\left\|\Delta \mu^{(1)}\right\| n_{b} k_{b}\right\rangle= \\
& \quad\left(k_{a}+k_{b}-1\right)\left\langle n_{a} k_{a}\left\|C^{(1)}\right\| n_{b} k_{b}\right\rangle\left[r^{(0)}\right]_{n_{a} k_{a} n_{b} k_{b}},
\end{aligned}
$$
where

$$
\left\langle k_{a}\left\|C^{(1)}\right\| k_{b}\right\rangle=
$$

$\left\{\begin{array}{l}(-1)^{j_{b}-1 / 2} \sqrt{2 j_{b}+1}\left(\begin{array}{lll}j_{a} & J_{b} & 1 \\ 1 / 2 & -1 / 2 & 0\end{array}\right) \text { for even } l_{a}+1+l_{b}, \\ 0 \text { for odd } l_{a}+1+l_{b},\end{array}\right.$

$$
\begin{aligned}
& {[r]_{n_{a} k_{a} n_{b} k_{b}}=\int_{0}^{\infty} r\left(P_{n_{a} k_{a}}(r) Q_{n_{b} k_{b}}(r)\right.} \\
& \left.+Q_{n_{a} k_{a}}(r) P_{n_{b} k_{b}}(r)\right) \mathrm{d} r, \\
& {\left[r^{(0)}\right]_{n_{a} k_{a} n_{b} k_{b}}=\int_{0}^{\infty} r^{(0)}\left(P_{n_{a} k_{a}}(r) P_{n_{b} k_{b}}(r)\right.} \\
& \left.\quad+Q_{n_{a} k_{a}}(r) Q_{n_{b} k_{b}}(r)\right) \mathrm{d} r .
\end{aligned}
$$

\subsection{Hyperfine interaction}

In relativistic framework, the hyperfine Hamiltonian is

$$
\widehat{H}_{h f s}=\sum_{i} T_{n}^{(k)}(j) T_{e}^{(k)}(i),
$$


where $T_{n}^{(k)}$ and $T_{e}^{(k)}$ are the spherical tensor operator of rank $k$, representing the nuclear and electron angular momentum, respectively, $j$ and $i$ designate the various protons and electrons, respectively.

The mean value of $\widehat{H}_{h f s}$ in a fine structure state $\left|J I F M_{F}\right\rangle$ is given to first order as

$$
\begin{aligned}
& \left\langle\widehat{H}_{h f s}\right\rangle=\sum_{i} \delta_{M_{F} M_{F^{\prime}}} \delta_{F F^{\prime}}(-1)^{F+J+I}\left\{\begin{array}{ccc}
F & J & I \\
k & I & J^{\prime}
\end{array}\right\} \\
& \quad \times\left\langle I|| T_{n}^{(k)} \| I\right\rangle\left\langle J\left\|T_{e}^{(k)}\right\| J^{\prime}\right\rangle,
\end{aligned}
$$

where $I$ is the nuclear spin and $F=J+I$ is the total angular momentum of the atom.

For the magnetic dipole case, $k=1$, while the in the electric quadrupole case $k=2$.

For seek of simplicity, we express the nuclear dipole moment in nuclear magneton $\mu_{n}$ units, the nuclear dipole moment $\mu_{I}$ can be written as

$$
\begin{gathered}
\mu_{I} \mu_{n}=\left\langle I M_{I}=I\left|T_{n 0}^{(k)}\right| I M_{I}=I\right\rangle= \\
\left(\begin{array}{ccc}
I & k=1 & I \\
-I & 0 & I
\end{array}\right)\left\langle I|| T_{n}^{(1)}|| I\right\rangle .
\end{gathered}
$$

On the other hand, the operator $T_{q}^{(1)}$ is given by [24, 25]:

$$
T_{q}^{(1)}=\sum t_{q}^{(1)}=-\sum_{j} i e \sqrt{\frac{8 \pi}{3}} \frac{1}{r_{j}^{2}} \boldsymbol{\alpha}_{j} \cdot Y_{1 q}^{(0)}\left(\widehat{r}_{j}\right),
$$

where $e$ is the absolute magnitude of the electron charge and $j$ denotes the $j$-th electron in the atom.

3.3.1. Calculation of the magnetic dipole hyperfine constant $A_{M D}$

The hyperfine constant $A_{M D}$ is related to the nuclear magnetic moment by

$$
A_{M D}=\mu_{n} \frac{\mu_{I}}{I}\left\langle J|| T^{(1)} \| J\right\rangle(J(J+1)(2 J+1))^{-1 / 2} .
$$

By means of Eq. (40), the hyperfine energy splitting $E_{M D}$ due to the magnetic dipole is

$$
E_{M D}=\frac{A_{M D}}{2}(F(F+1)-I(I+1)-J(J+1)),
$$

3.3.2. Calculation of the electric quadrupole constant $B_{E Q}$

The electric quadrupole constant $B_{E Q}$ is related to the nuclear quadrupole moment $Q$. The latter is given by

$$
\begin{gathered}
e Q=2\left\langle I, M_{I}=I\left|Q_{0}^{(2)}\right| I, M_{I}=I\right\rangle= \\
\left(\begin{array}{ccc}
I & k=2 & I \\
-I & 0 & I
\end{array}\right) 2\left\langle I\left\|Q_{0}^{(2)}\right\| I\right\rangle .
\end{gathered}
$$

Since $e$ is the charge and $Q$ is the operator for the proton, then $e Q$ represent the charge reparation in the nucleus and ( ) denotes 3-j symbol.

On the other hand, the operator $T_{q}^{(2)}$ is given by [26] as

$$
T_{q}^{(2)}=\sum t_{q}^{(2)}=\sum_{j} \frac{-e}{r_{j}^{3}} \sqrt{\frac{\pi}{(2 k+1)}} Y_{q k} .
$$

Here, the summation is carried out over all electrons of the atom.

The electric quadrupole hyperfine constant becomes

$$
\begin{aligned}
& B_{E Q}=2 e Q\left[\frac{2 J(2 J-1)}{(2 J+1)(2 J+2)(2 J+3)}\right]^{1 / 2} \\
& \quad \times\left\langle J\left\|T^{(2)}\right\| J\right\rangle .
\end{aligned}
$$

By means of Eq. (44), the hyperfine energy splitting $E_{E Q}$ due to the electric quadrupole is

$$
\begin{aligned}
& E_{E Q}=\frac{B_{E Q}}{8} \frac{(3 C(C+1)-4 J(J+1) I(I+1))}{J(2 J-1) I(2 I-1)}, \\
& C=F(F+1)-I(I+1)-J(J+1) .
\end{aligned}
$$

\section{Uncertainty evaluation in theoretical results}

The complete state of the theoretical results should include an estimate of the accuracy associated with these results. A priori, reporting theoretical results with their uncertainties helps us to make judgement about the quality of these results and facilitates meaningful comparison with other similar experimental ones or theoretical predictions.

The uncertainty of transition energy in spin-orbit coupling ( $L S$-coupling) is given by [27] as

$$
\delta E=\frac{\left|E_{\text {calc }}-E_{\exp }\right|}{E_{\exp }} .
$$

Consequently, the uncertainty in the theoretical values of the Landé $g$-factor $\delta g$ can be given by

$$
\delta g=\frac{\left|g_{\text {calc }}-g_{\exp }\right|}{g_{\exp }} .
$$

However, the uncertainty of the computed transition rates $A_{f i}$ is given by [28] as

$$
\delta \tilde{A}_{f i}=\frac{\left|A^{\prime}{ }_{f i}(l)-A_{f i}^{\prime}(v)\right|}{\max \left(A_{f i}^{\prime}(l), A_{f i}^{\prime}(v)\right)},
$$

where $A_{f i}^{\prime}(l)$ and $A_{f i}^{\prime}(v)$ are the energy scaled-length and velocity, respectively. These scaled are estimated by $[28]$ as

$$
\begin{aligned}
& A^{\prime}{ }_{f i}(l)=A_{f i}(l)\left(\frac{E_{\text {exp }}}{E_{\text {calc }}}\right)^{3}, \\
& A^{\prime}{ }_{f i}(v)=A_{f i}(v)\left(\frac{E_{\text {exp }}}{E_{\text {calc }}}\right) .
\end{aligned}
$$

\section{Numerical results and discussions}

The above equations are plugged in a power full General Relativistic Structure Package code called GRASP [29]. It is an integrated software package to predict various atomic spectroscopy parameters, such as atomic energy levels, oscillator strengths, radiative decay rates, hyperfine structure constants, the Landé $g$-factors, and specific mass shift parameters. These parameters are predicted by using a multiconfiguration Dirac-HartreeFock approach.

\subsection{Calculations of energy levels and prediction of oscillator strengths}

The energy levels of the intermediate Rydberg odd series $n d[1 / 2] 1 \quad(3 \leq n \leq 9)$ relative to $3 p^{6}{ }^{1} S_{0}$ are numerically calculated and tabulated in Table I. Since 
available experimental results provide a check on the accuracy of the calculation ones, the obtained results are compared with the experimental ones obtained by Minnhagen [30] with an uncertainty in the position of each level of $0.05 \mathrm{~cm}^{-1}$ as shown by Table I. Within the uncertainty in our theoretical results, this comparison shows a reasonable agreement between the theoretical and experimental results, therefore, we are confident of our energy levels listed in Table I. However, the small differences in the experimental and theoretical energies are due to the calculations of the central potential for radial orbitals and recoupling schemes of angular parts. Moreover, this difference might be due to the perturbation between $n d$ and $n d^{\prime}$ series.

TABLE I

Energy levels, dimensionless oscillator strengths $f_{i f}$, of the odd series $n d[1 / 2]_{1}(3 \leq n \leq 9)$ relative to $3 p^{6}{ }^{1} S_{0}$ of neutral argon atom $\operatorname{Ar}(\mathrm{I})$, as obtained in the length $\left(F_{l}\right)$ and velocity $\left(F_{V}\right)$ forms of the electric dipole operator in the Babushkin and Coulomb gauge, respectively.

\begin{tabular}{|c|c|c|c|c|c|}
\hline \multirow[b]{2}{*}{$n$} & \multicolumn{2}{|r|}{ Energy } & \multicolumn{2}{|c|}{ Oscillator strengths } & Ratio \\
\hline & $E_{\exp }\left[\mathrm{cm}^{-1}\right]$ & $E_{\text {theor }}\left[\mathrm{cm}^{-1}\right]$ & $F_{l}$ & $F_{V}$ & $F_{l} / F_{V}$ \\
\hline 3 & 111818.028 & $111823.09 \pm 4.527 \times 10^{-5}$ & 0.086414 & 0.095866 & 0.901 \\
\hline 4 & 118651.395 & $118647.46 \pm 3.318 \times 10^{-5}$ & 0.187570 & 0.207608 & 0.903 \\
\hline 5 & 121932.8477 & $121937.00 \pm 3.402 \times 10^{-5}$ & 0.147075 & 0.162141 & 0.907 \\
\hline 6 & 123467.9733 & $123470.10 \pm 1.721 \times 10^{-5}$ & 0.104600 & 0.115051 & 0.909 \\
\hline 7 & 124554.874 & $124557.93 \pm 2.452 \times 10^{-5}$ & 0.072790 & 0.079965 & 0.910 \\
\hline 8 & 125135.831 & $125135.35 \pm 3.808 \times 10^{-6}$ & 0.051377 & 0.056403 & 0.910 \\
\hline 9 & 125613.07 & $125614.17 \pm 8.769 \times 10^{-6}$ & 0.037159 & 0.040777 & 0.911 \\
\hline
\end{tabular}

The interaction between the excited electron and the electrons in the core is complicated by exchange effect. These complications are confined to the region of configuration space where the electron penetrates the ionic core. The behavior of stationary wave functions in this region varies slowly with the degree of excitation and common to the wave functions of many excited and ionized states in the vicinity of the ionization limits.

Table I shows that for excitation energies less than the first limit of ionization, there are many number of discrete levels forming perturbed Rydberg series. Using the accurate location of these levels, the corresponding oscillator strength $f_{i j}$ in the length $\left(F_{l}\right)$ and velocity $\left(F_{V}\right)$ forms of the electric dipole operator, respectively, were calculated. The obtained results are also tabulated in Table I. The fifth column of Table I shows the ratio $F_{l} / F_{v}$. The obtained results confirmed the fact that $F_{l}$ and $F_{v}$ are of the same order despite the fact that they are calculated in different gauge. On the other hand, our theoretically obtained results of the oscillator strength for the levels $3 d[1 / 2] 1$ is compared with those obtained by other researcher either experimentally [31-34] or theoretically $[35,36]$. Table II shows these comparisons. Westerveld and co-workers [31] measured experimentally the oscillator strengths using self-absorption of resonance radiation for fourteen resonance transitions of neon and argon. The measurements on argon transitions were carried out in the first order spectrum. The problem with these transitions is the overlap with lines in second and third order. However, Westerveld and co-workers arranged their experimental setup in order to eliminate any contribution of these higher order lines in the first order spectrum of argon. Therefore, these higher order lines contributions are responsible of the slight difference between our results and the Westerveld ones.

TABLE II

Comparison of the oscillator strength $f_{i j}$ for the $3 p^{6}{ }^{1} S_{0} \rightarrow n d[1 / 2]_{1}$ transition array.

\begin{tabular}{c|c|c|c|c|c|c}
\hline \hline \multirow{3}{*}{$n$} & \multicolumn{4}{|c|}{ Experimental } & \multicolumn{2}{c}{ Theoretical } \\
\cline { 2 - 7 } & \multicolumn{2}{|c|}{ Present work } & $\begin{array}{c}\text { Electron } \\
\text { impact } \\
\text { method [30] }\end{array}$ & $\begin{array}{c}\text { Chan } \\
\text { et al. } \\
{[31,32]}\end{array}$ & $\begin{array}{c}\text { Lee and } \\
\text { Lu [33] }\end{array}$ & $\begin{array}{c}\text { Lee } \\
{[34]}\end{array}$ \\
\cline { 2 - 7 } & $F_{l}$ & $F_{V}$ & $f_{i j}$ & $f_{i j}$ & $f_{i j}$ & $f_{i j}$ \\
\hline 3 & 0.086414 & 0.095866 & 0.0929 & 0.0914 & 0.092 & 0.092
\end{tabular}

Unfortunately, these are the only levels that we found in the literature. The lack of experimental results of oscillator strengths and transition rates for the levels in question is due to the excitation energies. These levels are autoionized and their absorption spectra are increasingly overlapping and difficult to resolve as the excitation energy approaches the limits of ionization. The lack of experimental results for both oscillator strengths and transition rates suggests that more appropriate theoretical models are necessary for spectrum calculations that included the highly excited states as well as the ground states.

\subsection{Calculation of the radiative transition rates}

We calculated the radiative transition rates $A_{f i}$ (in $\mathrm{s}^{-1}$ ) of the odd series $n d[1 / 2]_{1}(3 \leq n \leq 9)$ relative to $3 p^{6}{ }^{1} S_{0}$ of neutral argon atom $\operatorname{Ar}(\mathrm{I})$, as obtained in the length $A_{f i}(l)$ and velocity $A_{f i}(v)$ forms of the electric dipole operator. The obtained results are tabulated 
in Table III. We were unable to compare these results with other results published either by other researchers or NIST web site. To the best of our knowledge, these transition rates are calculated for the first time.

\section{TABLE III}

Radiative transition rates $A_{f i}\left[\mathrm{~s}^{-1}\right]$ of the odd series $n d[1 / 2]_{1}(3 \leq n \leq 9)$ relative to $3 p^{6}{ }^{1} S_{0}$ of neutral argon atom $\operatorname{Ar}(\mathrm{I})$, as obtained in the length $A_{f i}(l)$ and velocity $A_{f i}(V)$ forms of the electric dipole operator in the Babushkin and Coulomb gauge, respectively.

\begin{tabular}{c|c|c|c}
\hline \hline$n$ & $A_{f i}(l)$ & $A_{f i}(V)$ & $\delta \tilde{A}_{f i}$ \\
\hline 3 & $2.40732 \times 10^{8}$ & $2.66922 \times 10^{8}$ & 0.09820 \\
4 & $2.07298 \times 10^{8}$ & $2.29444 \times 10^{8}$ & 0.09640 \\
5 & $1.72738 \times 10^{8}$ & $1.90433 \times 10^{8}$ & 0.09298 \\
6 & $1.26840 \times 10^{8}$ & $1.39513 \times 10^{8}$ & 0.09086 \\
7 & $8.99551 \times 10^{7}$ & $9.88217 \times 10^{7}$ & 0.08981 \\
8 & $6.42726 \times 10^{7}$ & $7.05599 \times 10^{7}$ & 0.08910 \\
9 & $4.68733 \times 10^{7}$ & $5.14367 \times 10^{7}$ & 0.08873
\end{tabular}

\subsection{Calculation of Landé g-factor and the hyperfine structure constants}

The relativistic Landé $g$-factor is calculated for the odd series $n d[1 / 2]_{1}(3 \leq n \leq 9)$ relative to the ground level $3 p^{6}{ }^{1} S_{0}$. The obtained results are tabulated in Table IV. As there is no theoretical data of the Landé $g$-factor, many experimental ones have been left without comparison with the theoretical ones. However, our results are compared to those obtained, experimentally by Salah [2], Chenevier and Moskowitcz [37] or theoretically by Aymar and Schweighofer [7] as well as NIST. The comparison shows an acceptable agreement between these results. The Landé $g$-factor for the levels $3 d[1 / 2]_{1}$, $4 d[1 / 2]_{1}, 5 d[1 / 2]_{1}, 10 d[1 / 2]_{1}$ and $11 d[1 / 2]_{1}$ are not measured by Salah, while he measured the Landé $g$-factor for the levels $6 d[1 / 2]_{1}, 7 d[1 / 2]_{1}, 8 d[1 / 2]_{1}$ and $9 d[1 / 2]_{1}$ by using optogalvanic atomic laser spectroscopy. The uncertainty of his results is estimated to be 0.02 . On the other side, Chenevier and Moskowitz [37] measured experimentally the Landé $g$-factor for the levels $3 d[1 / 2]_{1}$ using optically detected magnetic resonance technique. The theoretical value of this level is determined by Aymar and Schweighofer [7]. Since there is no other experimental or theoretical data for the levels under consideration available in the literature, our theoretical results could be used to determine the hyperfine constants in cross level experiment [38-40]. They also provide a sensitive test to the different part of atomic wave functions from those decisive to the hyperfine constant and the oscillator strengths.

The magnetic dipole $A_{M D}$ and the electric quadrupole $B_{E Q}$ hyperfine constants are calculated by GRASP. The obtained results are tabulated in Table $\mathrm{V}$.

It is worth to mention that the theoretical results of Landé $g$-factor for the odd series $n d[1 / 2]_{1}(3 \leq n \leq 9)$ and the hyperfine structure constants are reported here for the first time.
TABLE IV

Relativistic Landé $g$-factor for the odd Rydberg series $n d[1 / 2]_{1}(3 \leq n \leq 9)$ relative to $3 p^{6}{ }^{1} S_{0}$ of neutral argon atom.

\begin{tabular}{c|c|c|c|c|c}
\hline \hline & This & \multicolumn{2}{|c|}{ Exp. work } & \multirow{2}{*}{ Theor. work } \\
\cline { 3 - 4 }$n n$ & work & {$[2]$} & {$[37]$} & NIST & {$[7]$} \\
\hline 3 & $1.45930 \pm 0.01962$ & & $1.4885(20)$ & & 1.481 \\
4 & $1.42157 \pm 0.03097$ & & & 1.467 & \\
5 & 1.38549 & & & & \\
6 & $1.21278 \pm 0.00591$ & 1.22 & & & \\
7 & $1.34326 \pm 0.00997$ & 1.33 & & & \\
8 & $1.21507 \pm 0.00405$ & 1.22 & & & \\
9 & $1.32564 \pm 0.01972$ & 1.30 & & & \\
\end{tabular}

TABLE V

The hyperfine structure constants for the odd Rydberg series $n d[1 / 2]_{1}(3 \leq n \leq 9)$ relative to $3 p^{6}{ }^{1} S_{0}$ of neutral argon atom.

\begin{tabular}{c|c|c}
\hline \hline$n$ & $A_{M D}[\mathrm{MHz}]$ & $B_{Q E}[\mathrm{MHz}]$ \\
\hline 3 & -19.98789 & -30.69076 \\
4 & -13.77403 & -24.18999 \\
5 & 33.07344 & -28.41008 \\
6 & 44.36763 & -31.02961 \\
7 & 50.88277 & -32.60004 \\
8 & 54.78067 & -33.56238 \\
9 & 57.23293 & -34.176258
\end{tabular}

\section{Conclusion}

A fully relativistic approach based on the Dirac equation is used to calculate the spectroscopic data of the odd series $n d[1 / 2]_{1}(3 \leq n \leq 9)$ relative to the ground level $3 p^{6}{ }^{1} S_{0}$. These series are known as intermediate or quasi-Rydberg series [41, 42]. The energy levels of this series are calculated. The obtained results are judged by comparing them with the experimental ones available in the literature. Within the uncertainty in our theoretical results, the agreement between our results and the experimental ones was found to be reasonable. Based on this agreement, the oscillator strengths $f_{i j}$, the radiative transition rates $A_{i j}$, the Landé $g$-factor, and the magnetic dipole $A_{M D}$ and the electric quadrupole $B_{E Q}$ hyperfine constants for the levels in question are predicted. The obtained results of the oscillator strengths and Landé $g$ factor are compared with the available experimental and theoretical ones. A fairly good agreement is found.

The Landé $g$-factor is a measure of the magnetic sensitivity of atomic levels. Heretofore, the experimental values for Landé $g$-factor of argon neutral atoms are far from being complete. Consequently, our results in this paper could be useful to investigations for some radiative parameters in argon atoms.

Our new, fairly complete, set of precise and reliable theoretical results could be of great interest for both experimental and theoretical research on neutral argon atoms. These results should stimulate further theoretical work in the atomic spectroscopy field. 


\section{References}

[1] M. Hanif, M. Aslam, R. Ali, A. Nadeem, M. Riaz, S.A. Bhatti, M.A. Baig, J. Phys. B At. Mol. Opt. Phys. 33, 4647 (2000).

[2] W. Salah, Nucl. Instrum. Methods Phys. Res. B 196, 25 (2002).

[3] N.K. Piracha, M.A Baig, S.H Khan, B. Suleman, J. Phys. B At. Mol. Opt. Phys. 30, 1151 (1997).

[4] X. Husson, J.P. Grandin, J. Phys. B At. Mol. Opt. Phys. 12, 3649 (1979).

[5] H. Abu Safia, J.-M. Margerie, J. Phys. B At. Mol. Opt. Phys. 16, 927 (1983).

[6] M. Oba, K. Akaoka, M. Miyabe, I. Wakaida, Eur. Phys. J. D 10, 349 (2000).

[7] M. Aymar, M.G. Schweighofer, Physica 67, 585 (1973).

[8] K. Yoshino, J. Opt. Soc. Am. 60, 1220 (1970).

[9] K.T. Lu, U. Fano, Phys. Rev. A 2, 81 (1970).

[10] G. Racah, Phys. Rev. 62, 438 (1942).

[11] R.D. Cowan, The Theory of Atomic Structure and Spectra, University of California, Berkeley, CA 1981.

[12] M. Aymar, M. Coulombe, At. Data Nucl. Data Tables 21, 537 (1978).

[13] M. Aymar, O. Robaux, C. Thomas, J. Phys. B At. Mol. Opt. Phys. 14, 4255 (1981).

[14] A.R. Edmonds, Angular Momentum in Quantum Mechanics, Princeton University Press, Princeton, NJ 1996.

[15] I.P. Grant, J. Phys. B 7, 145 (1974).

[16] J.D. Jackson, Am. J. Phys. 70, 917 (2002).

[17] G.S. Adkins, Phys. Rev. D 36, 1929 (1987).

[18] F.A. Babushkin, Opt. Spectrosc. 13, 77 (1962).

[19] F.A. Babushkin, Acta Phys. Pol. 25, 749 (1964).

[20] F.A. Babushkin, Opt. Spectrosc. 19, 1 (1965).

[21] A. Messiah, Quantum Mechanics, Vol. 2, North Holland, Amsterdam 1965, p. 927.

[22] A.J. Akhiezer, V.B. Berestetskii, Quantum Electrodynamics, Interscience, New York 1965, Ch. 8, Sect. 50.2 .
[23] N.C. Pyper, I.P. Grant, N. Beatham, Comput. Phys. Commun. 15, 387 (1978).

[24] I. Lindgren, A. Rosen, Case Stud. At. Phys. 4, 93 (1975).

[25] I. Lindgren, A. Rosen, Case Stud. At. Phys. 4, 197 (1975).

[26] J.P. Declaux, Comput. Phys. Commun. 9, 31 (1975).

[27] C. Fischer, Phys. Scr. T 134, 014019 (2009).

[28] J. Ekman, M.R. Godefroid, H. Hartman, Atoms 2, 215 (2014).

[29] P. Jönsson, G. Gaigalas, J. Bieron, C. Froese Fischer, I.P. Grant, Comput. Phys. Commun. 184, 2197 (2013).

[30] L. Minnhagen, J. Opt. Soc. Am. 63, 1185 (1973).

[31] W.B. Westerveld, Th.F.A. Mulder, J. van Eck, J. Quant. Spectrosc. Radiat. Transfer 21, 533 (1979).

[32] S.L. Wu, Z.P. Zhong, R.F. Feng, S.L. Xing, B.X. Yang, K.Z. Xu, Phys. Rev. A 51, 4494 (1995).

[33] W.F. Chan, G. Cooper, X. Guo, G.R. Burton, C.E. Brion, Phys. Rev. A 46, 149 (1992).

[34] W.F. Chan, X. Guo, G.R. Burton, C.E. Brion, Phys. Rev. A 48, 858 (1993).

[35] C.M. Lee, K.T. Lu, Phys. Rev. A8, 1241 (1973).

[36] C.M. Lee, Phys. Rev. A10, 584 (1974).

[37] M. Chenevier, P.A. Moskowitz, J. Phys. B 35, 401 (1974).

[38] X. Husson, J.P. Grandin, J. Phys. B At. Mol. Opt. Phys. 11, 1393 (1978).

[39] G. Binet, X. Husson, J.P. Grandin, J. Phys. Lett. 44, 151 (1983).

[40] H. Abu Safia, J.P. Grandin, X. Husson, J. Phys. B At. Mol. Opt. Phys. 14, 3363 (1981).

[41] J. Wolnikowski, A. Bielski, R.S. Trawiński, J. Szudy, Phys. Scr. 47, 186 (1993).

[42] R.S. Trawiński, A. Bielski, J. Szudy, J. Wolnikowski, Acta Phys. Pol. A 86, 333 (1994). 